\title{
Interleukine 10 et cellules B centrofolliculaires
}

L'IL10 est produite par les cellules $\mathrm{T}$ de type $\mathrm{TH} 2$, parallèlement à l'IL4 et l'IL5, mais également par les monocytes et les cellules B [1-4]. Cette lymphokine, individualisée initialement par sa capacité d'inhiber la sécrétion de cytokine produite par les cellules de type THl, c'està-dire l'IL2 et l'interféron $\gamma$, est également capable d'inhiber la prolifération de clones $\mathrm{T}$ spécifiques d'un antigène [5]. L'effet inhibiteur de l'ILl0 est expliqué principalement par son action sur les monocytes, marquée par une diminution de la production de cytokines et de l'expression des antigènes d'histocompatibilité de classe II, ne permettant plus une présentation efficace de l'antigène [6]. Par ailleurs, l'IL10 est également un facteur de croissance pour les mastocytes et les cellules $T$. Cette cytokine possède, enfin, des effets remarquables sur les cellules B dont elle favorise la prolifération et la différenciation, lorsque la cellule B est stimulée par un antigène, ou lorsque la molécule CD40 s'associe à son ligand présent sur les cellules $\mathrm{T}$ stimulées [7]. Chez la souris, l'IL10 est capable d'augmenter la viabilité des cellules B [8]. Les propriétés de l'ILl0 sur les cellules B nous ont permis d'émettre l'hypothèse qu'elle pourrait intervenir dans la sélection de cellules B spécifiques d'un antigène lors de la réponse immune dépendante des cellules $\mathrm{T}$. Le centre germinatif des ganglions lymphoïdes est au cœur de cette réponse immune puisque coexistent, au sein d'un follicule, la cellule dendritique présentatrice de complexes antigène-anticorps, des tre germinatif se développe en moins de dix jours après l'introduction d'un antigène; chaque centre germinatif d'un ganglion est ensemencé par un petit nombre de cellules $\mathrm{B}$ (une à quatre cellules) qui se divisent activement pour donner en quelques jours l'aspect du centre germinatif secondaire où prolifèrent de manière intense des centroblastes. Seuls quelques-uns de ces immunoblastes, exprimant des immunoglobulines de membrane ayant une forte affinité pour l'antigène, survivront, alors que la majorité de ces cellules B est destinée à mourir, faute de recevoir une stimulation adéquate [9]. Le processus de diversification somatique des molécules d'immunoglobulines est, à l'évidence, l'un des facteurs essentiels de cette sélection. Ainsi, si l'on sépare les immunoblastes d'un centre germinatif secondaire, les cellules $\mathrm{B}$ meurent dans leur majorité, au bout de quelques heures ou quelques jours de culture, par apoptose. Ce phénomène de mort cellulaire peut être prévenu in vitro par la stimulation des cellules B par des anticorps anti-immunoglobulines ou antiCD40, ou en présence d'lLl $\alpha$ associée à la molécule CD23 soluble [10, 11]. Dans ces conditions de culture, la survie des cellules centrofolliculaires est parallèle à l'induction de la synthèse de la protéine $\mathrm{Bcl} 2$ qui est effectivement susceptible de prévenir certaines formes d'apoptose $(\mathrm{m} / \mathrm{s}$ $n^{\circ} 2$, vol. 10, p. 208). En effet, à l'état physiologique, les cellules centrofolliculaires expriment l'ARN codant pour Bcl2 mais ne synthétisent pas, ou en quantité extrêmement faible, cette protéine.
Nous avons montré que l'ILl0 est une cytokine essentielle à la survie des cellules centrofolliculaires; elle induit la synthèse de la protéine Bcl2 dans ces cellules et les protège de l'apoptose habituellement constatée in vitro [12]. Le rôle essentiel de l'ILl0 dans la prévention de l'apoptose des cellules centrofolliculaires est démontré par l'utilisation d'anticorps neutralisant l'activité biologique de cette cytokine: en présence de ces anticorps, l'induction de la protéine $\mathrm{Bcl} 2$ par d'autres cytokines comme l'IL2 et l'IL4 est inhibée. Plusieurs autres types de cellules présentes dans les centres germinatifs, tels les monocytes et les cellules $\mathrm{T} \mathrm{CD} 4+$, sont potentiellement capables de synthétiser de l'ILl0. Cependant, les cellules B stimulées par un antigène pourraient produire directement de l'ILl 0 induisant, par une action autocrine, l'expression de la protéine $\mathrm{Bcl} 2$ et la survie des cellules B spécifiques qui pourront quitter le ganglion et rejoindre la moelle osseuse ou le compartiment des cellules B «mémoires".

Cet effet de l'IL10 sur la survie des lymphocytes B pourrait jouer un rôle dans la physiopathologie de certaines hémopathies lymphoïdes et dans les maladies auto-immunes. En effet, cette cytokine pourrait être impliquée dans le développement de certains lymphomes folliculaires, en l'absence de réarrangement du gène bcl2[13] et favoriser l'apparition d'auto-anticorps, comme cela est observé dans les modèles de souris transgéniques surexprimant le gène $b c l 2$ [14].

J.C.B. Y.L. 
1. Fiorentino DF, Bond MW, Mosmann TR. Two types of mouse $\mathrm{T}$ helper cells. IV. Th2 clones secrete a factor that inhibits cytokine production by Thl clones. J Exp Med 1989; 170: 2081-95.

2. de Waal Malefyt R, Abrams J, Bennett B, Figdor CG, de Vries JE. Interleukin 10 (IL10) inhibits cytokine synthesis by human monocytes : an autoregulatory role of IL10 produced 3. Burdin N, Péronne C, Banchereau J, Rousset F. Epstein-Barr virus transformation induces B lymphocytes to produce human interleukin 10. J Exp Med 1993; 177 : 295-304. 4. Goldman M, Velu T. L'interleukine 10, une nouvelle cytokine immunosuppressive et anti-inflammatoire. médecine/sciences $1993 ; 9$. 453-5.

5. Fiorentino DF, Zlotnik A, Vieira, et al. IL10 acts on the antigen presenting cell to inhibit cytokine production by Thl cells. J Immunol 1991 ; 146: 3444-51.

6. de Waal Malefyt R, Haanen J, Spits H, et al. Interleukin 10 (IL10) and viral IL,10 by monocytes. J Exp Med 1991 ; 174 : 1209-20. strongly reduce antigen-specific human $\mathrm{T}$ cell proliferation by diminishing the antigenpresenting capacity of monocytes via downregulation of class II major histocompatibility complex expression. I Exp Med 1991; 174 : 915-24

7. Rousset F, Garcia E, Defrance T, Péronne C, Vezzio N, Hsu DH, Kastelein R, Moore KW, Banchereau J. Interleukin 10 is a potent growth and differentiation factor for activated human B lymphocytes Proc Natl Acad Sci USA 1992; 89: 1890-3.

8. Go NF, Castle BE, Barrett R, et al. Interleukin 10, a novel B cell stimulatory factor: unresponsiveness of $\mathrm{X}$ chromosome-linked immunodeficiency B cells. J Exp Med 1990; 172: 1625-31

9. Liu YJ, Mason DY, Johnson GD, et al. Germinal center cells express Bcl-2 protein after activation by signals which prevent their entry into apoptosis. Eur I Immunol 1991; 21 : 1905-10.

10. Liu YJ, Joshua DE, Williams GT,
Smith CA, Gordon J, MacLennan. Mechanism of antigen-driven selection in germinal centres. Nature 1989 ; 342 : 929-31.

11. Liu YJ, Cairns JA, Holder MJ, et al. Recombinant 25-kDa CD23 and interleukin $1 \alpha$ promote the survival of germinal center $B$ cells : evidence for bifurcation in the development of centrocytes rescued from apoptosis. Eur J Immunol 1991; 21 : 1107-14.

12. Levy Y, Brouet JC. Interleukin 10 prevents spontaneous death of germinal center B cells by induction of the Bcl-2 protein. J Clin Invest 1994; 93 : 424-8.

13. Pezzella F, Tse AGD, Cordell JL, et al. Expression of the $\mathrm{Bcl}-2$ oncogene protein is not specific for the 14-18 chromosomal translocation. Am J Pathol 1990; 137 : 225-32.

14. Strasser A, Whittingham S, Vaux DL, Bath ML, Adams JM, Cory S, Harris AW. Enforced Bcl-2 expression in B-lymphoid cells prolongs antibody responses and elicits autoimmune disease. Proc Natl Acad Sci USA $1991 ; 88: 8661-5$.

\section{I BRÈVE}

Le diabète insipide néphrogénique autosomique récessif est dû à des mutations du gène de l'aquaporine 2. Le diabète insipide néphrogénique (NDI) lié à l'X est caractérisé par la résistance du canal collecteur rénal et de l'endothélium vasculaire aux effets des analogues $V_{2}$ de la vasopressine (AVP) et par la présence de mutations du gène codant pour le récepteur V2 de l'AVP. Mais il existe des cas de NDI non liés à l'X, apparemment autosomiques récessifs, caractérisés par l'absence de réponse du canal collecteur rénal à l'AVP, alors que la réponse endothéliale est normale. Une anomalie d'un transporteur spécifique de l'eau était soupçonnée et sa recherche très active depuis la première description d'une aquaporine, protéine-canal de l'eau identifiée dans le globule rouge $(\mathrm{m} / \mathrm{s}$ $n^{\circ}$ 3, vol. 9, p. 334). Après avoir cloné l'ADNc de l'aquaporine du tube collecteur du rein de rat, Sasaki et al. (Osaka, Japon) en ont équivalente du rein humain [1]. Ils ont criblé une banque d'ADNc de rein humain et ont isolé un clone fait de même pour l'aquaporine
d'ADNc codant pour une protéine de 271 acides aminés qui a $91 \%$ d'identité avec l'aquaporine du canal collecteur de rat. En Northern blot, l'expression de l'ARNm prédomine dans la médullaire rénale et en immunocytochimie, la fixation se limite aux cellules du canal collecteur, et plus spécialement au domaine apical. L'étude de l'expression dans des ovocytes de Xenopus confirme que cette aquaporine agit bien comme un canal de l'eau. Sa masse moléculaire est de 29 kDA. Le gène de cette aquaporine est localisé sur le chromosome 12q13. Ce gène devenait un excellent gène candidat pour le diabète insipide néphrologique congénital héréditaire, non-lié à l'X (voir $\mathrm{m} / \mathrm{s}$, $n^{\circ} 12$, vol. 9, p. 1428). Deen et al. [2] (Nimègues, Pays-Bas), étudiant un tel cas, ont comparé les séquences du gène normal aux séquences des quatre exons du gène du malade amplifiés à partir de l'ADN génomique, et identifié deux mutations ponctuelles hétérozygotes, l'une dans l'exon 3 (R187c), l'autre dans l'exon 4 (S216P), provenant respectivement du père et de la mère; le malade étudié est un hétérozygote composite, porteur de deux mutations récessives du gène de l'aquaporine-2. Les auteurs ont cloné les ADNc, normal et mutant, dans un vecteur d'expression et montré que leur transcription in vitro produit des protéines de $29 \mathrm{kDa}$. La fonction des canaux a été étudiée après injection dans des ovocytes de xénope des ARNc produits par transcription in vitro: les ARN mutant codent pour une protéine dont la fonction est réduite d'un facteur 10 par rapport à celle de l'aquaporine-2 normale. En revanche, la fonction du canal exprimé après micro-injection simultanée des ARN mutant et normal est normale. L'aquaporine-2 est bien, chez l'homme, le canal de l'eau, contrôlé par l'AVP; le diabète insipide néphrogénique peut être la manifestation de mutations survenant à la première et à la dernière étape du contrôle l'antidiurèse par l'AVP, soit dans le récepteur de l'AVP, soit dans l'aquaporine elle-même.

[1. Sasaki S, et al. I Clin Invest 1994; 93: 1250-6.]

[2. Deen PMT, et al. Science 1994 ; 264: 92-5.]
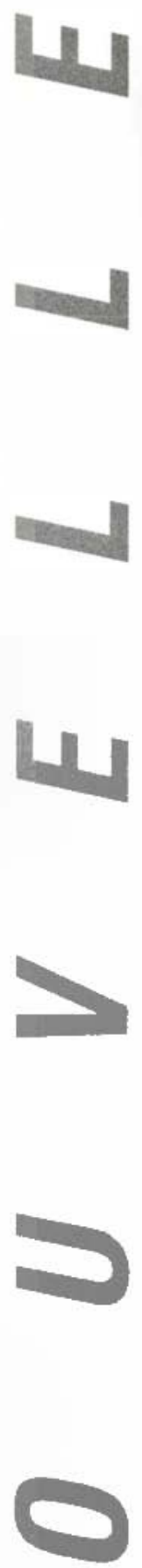

$\mathrm{m} / \mathrm{s} \quad n^{\circ} \quad 6-7$ vol. 10, juin-juillet 94 